\title{
Review of the Distributed Computing and EMR: Can EDI Solve the Problem?
}

\author{
Priti B. Kalode \\ PCD ICSR, VMV College Campus, Wardhaman \\ Nagar \\ Nagpur - 440008
}

\author{
Sajid Khan \\ Prof. Ram Meghe Institute of Technology \& \\ Research, Amravati
}

\begin{abstract}
The impact of distributed computing in healthcare is just beginning to be realized. The goal of wide scale integration of electronic medical records while meeting stringent security, confidentiality and privacy requirements for the patient is a grand-challenge scale problem of global proportions with farreaching social benefits. Healthcare organizations have to protect private information pertaining to the individuals they serve. As more and more healthcare organizations implement computer-based Enterprise Resource Planning (ERPs), telemedicine, EDI, data warehouses and other network-based information systems, information security in healthcare gains importance more than ever before.
\end{abstract}

In this paper we are proposing two clinical services based systems, the ontology based data gleaning system, the gleaned data is sent to which has an in-built generalization privacy technique and the agent-based intelligent decision support system.

\section{Keywords}

gleaning systems, distributed computing, e-medicine, ehealth, ontological approach, EMR

\section{INTRODUCTION}

Information in the Healthcare domain is enormously complex, covering many different types of data. Patient administration, organisational information clinical data and lab oratory/pathology data are different but must be compensated. Add to this the integration of all of these areas, and storage in Electronic Health Records. As a result of this diversity and richness of the data, and also due to the fragmented nature of Health Informatics implementation and research efforts, many different models have been designed to represent information in this field [1].

There is a growing need for sharing patient records among health care providers and their associated facilities, in order to provide the best care and clinical outcomes for patients. Due to the increasing use of information technologies in health care industries, patient records are often produced and shared in an electronic form. In distributed computing systems (e.g. client-server systems or today's multi-tier systems) data is stored on many computers across an enterprise and even outside of the enterprise. The results of data being stored in and transmitted to numerous places (e.g. laptop, PDA, mobile phones) are massive and information system professionals recognized that data security would be an issue that had to be addressed with new solutions and new technologies [2].

Data confidentiality is most commonly associated with security and is easy to understand. Healthcare organizations must protect all confidential data so that it does not get disclosed either accidentally or maliciously [3]. There have been several instances when health information about a patient has been leaked. Such disclosure, whether of a celebrity's health data or a private citizen's health data, can ruin a person's career, insurability or even his life. Thus, using technology and policies to protect the confidentiality of electronic health care information is a must.

\section{CURRENT SCENARIO AND ASSOCIATED PROBLEMS}

The current way of developing and using healthcare information store system has lead to a chaotic state of affairs due to the following reasons [4]:

- They have been developed independently and do not easily interoperate with each other.

- They follow their own convention of creating, maintaining, and storing Electronic Health Records (EHRs) of patients.

- If a patient is treated at different hospitals at different instances, different EHRs are generated and stored for the same patient by the two different information systems in use at two hospitals. A single HER for an individual is desirable irrespective of his/her time and place of treatment.

- $\quad$ Each system has its own way of creating and managing its storage of EHRs. Obviously, such an information store is based on both relational database technology, due to its ubiquity and maturity in managing large volume of information, and media storage software, for example Xray picture archiving. Different systems use different rational database and different media storage software making data transfer across systems impossible/incontinent.

Most of the existing systems use centralized storage, leading to limited scalability and poor reliability (single point of failure).

As a result, it has become difficult to exchange EHRs across different systems and to have a unified information system to delay with one HER per individual, irrespective of the time and place of treatment of an individual.

Distributed systems have been in the mainstream of high performance computing with the increasing computing power of computers. They have been primarily used for solving comprehensive application problems such as parallel 
rendering, weather modelling, nuclear simulations, and data mining.

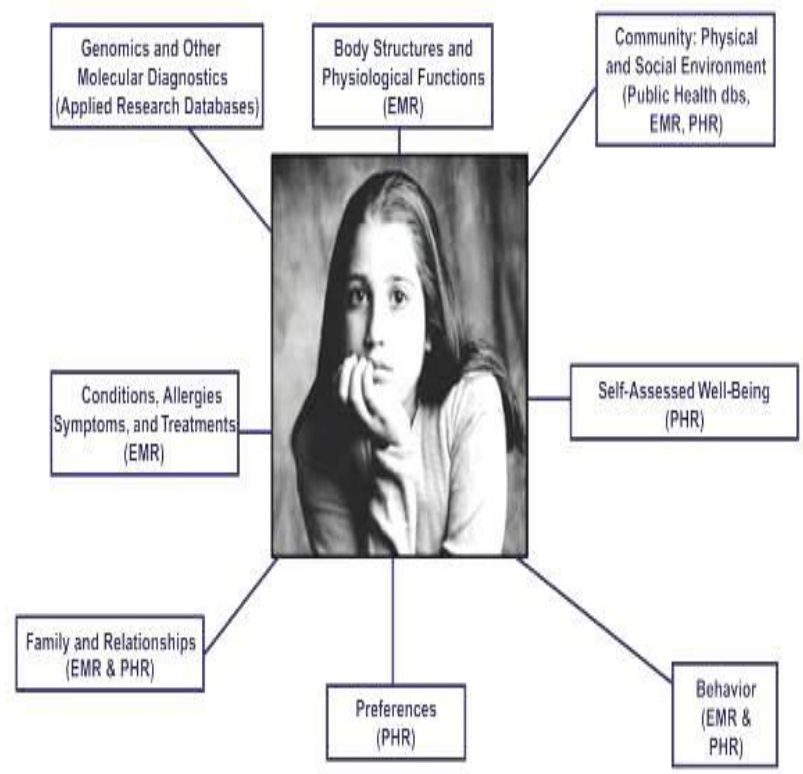

Fig.1

\section{REENGINEERING ENVIRONMENT}

Reengineering is the process of transforming an existing software system into a new form that can then be adapted to new purposes. The reengineering process generally entails the activities of analysing the system to extract and understand its design, assessing the problems, and migrating the system to new or transformed designs and implementations. This process is iterative, and can benefit greatly from tools to support in manipulating, navigating and analysing models of software.

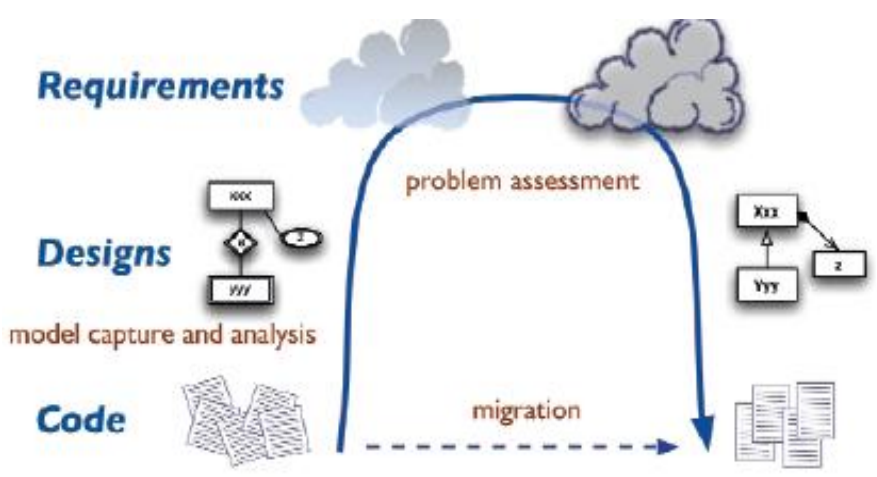

Figure 2: The Reengineering Process

\section{ONTOLOGY BASED DATA GLEANING SYSTEM}

The software development process is requirement analysis and design phase. It has become less common to develop new software from scratch. Instead, software is developed by adapting and/or combining existing re-usable software architectures.

In this research, we focus on semantic processing of requirements and re-usable parts by using ontology techniques. We are developing techniques that enable us to elicit semantically correct requirements and to select the implementation structures that are semantically suitable for those requirements. We have an ontology system whose concrete structure is the thesaurus of domain-specific words for each problem domain. In our new methodology, requirements are elicited based on the ontology, or more concretely, the ontology system guides the analysts' activities to elicit requirements. As a result, the meaning of the elicited requirements can be represented with a set of relevant words included in the ontology system. Re-usable parts are also semantically related to the words in the ontology system; that is, the meaning of each re-usable part is provided by the ontology system. Consequently, we have a kind of database of re-usable parts, including their semantic information. The selection of re-usable parts is performed by means of word matching in the ontology system. The re-usable parts are then adapted so as to satisfy the requirements and are integrated into a final product.

Our ontology system has two layers; one for requirements elicitation and the other for re-usable parts. By establishing relationships between the two layers, the ontology system can play a role in bridging gaps between a requirements specification and an architectural design at a semantic level.

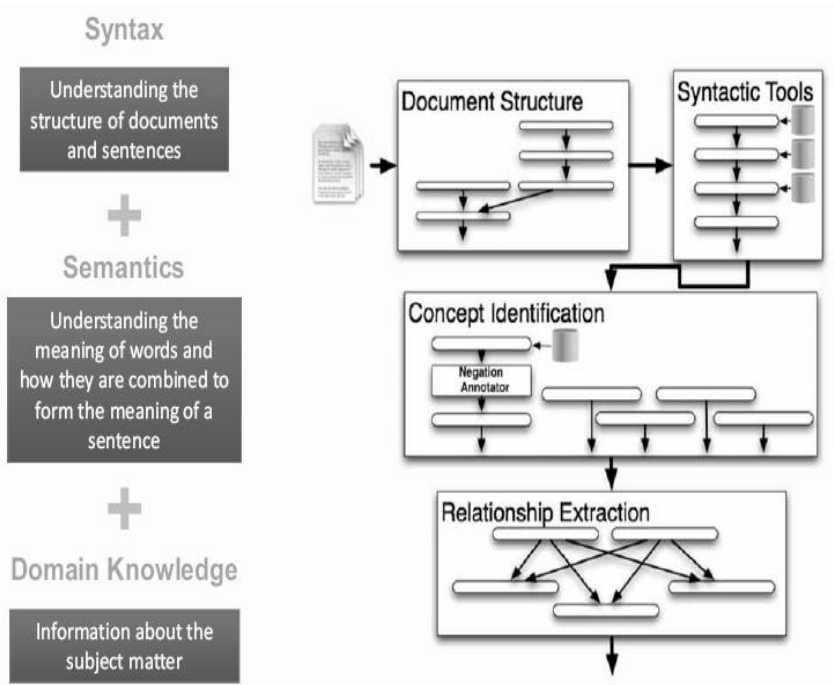

Figure 3: Making Sense of unstructured data using Natural Language Processing (NLP)

\section{SEMANTIC INTEROPERABILITY AND STANDARDS}

Right now the IT systems we have in use are essentially transactional systems that support actions such as ordering a lab test or documenting the administration of a medication, rather than cognitive support systems that help clinicians sort through data to think more clearly about critical health care decisions". Only integrated individual (personal) health records are seen to have 'true transformative potential to strengthen healthcare consumers' ability to manage their own health care'. Architecture is the conceptual design of the system. Systems inter-operate if their architectures are similar enough that functions that execute on one system execute identically (or nearly identically) on another system. Shared methods refer to the processes and procedures that a system performs. To ensure interoperability, these operations must be 
capable of being performed identically at any point in the network, regardless of implementation. A shared framework is a shared set of goals and strategies. One must agree on a shared set of goals and approaches to implementation.

Semantic: The ability to interpret, and, therefore, to make effective use of the information so exchanged.

- $\quad$ Shared data types (types of data exchanged)

- $\quad$ Shared terminologies (common vocabulary)

- $\quad$ Shared coding (standard encodings)

Shared data types refer to the types of data exchanged by systems. Interoperability requires that systems share data types on many different levels, including messaging formats (e.g. XML, ASCII), and programming languages (e.g. integer, string).

Shared terminologies refer to establishing a common vocabulary for the interchange of information. Standardized terminology is a critical requirement for healthcare applications to ensure accurate diagnosis and treatment, and has led to developing standards such as SNOMED-CT.

\section{IN-BUILT GENERALIZATION PRIVACY TECHNIQUE}

Information becomes sensitive when they are specific to a small number of individuals. Data mining, on the other hand, typically makes use of information shared by some minimum number of individuals to ensure a required statistical significance of patterns. As such, sensitive information are to be discarded for reliable data mining. This observation motivates us to apply the requirement of an intended data mining task to identify useful information to be released, therefore, sensitive information to be masked. This approach, called data mining based privacy protection, turns data mining from a threat into a solution to privacy protection.

The well-known privacy-preserved data mining modifies existing data mining techniques to randomized data. Here, we study data mining as a technique for masking data therefore, termed data mining based privacy protection. This approach incorporates partially the requirement of a targeted data mining task into the process of masking data so that essential structure is preserved in the masked data. The idea is simple but novel: we explore the data generalization concept from data mining as a way to hide detailed information, rather than discover trends and patterns. Once the data is masked, standard data mining techniques can be applied without modification. Our work demonstrated another positive use of data mining technology: not only can it discover useful patterns, but also mask private information.

\section{THE AGENT-BASED INTELLIGENT DECISION SUPPORT SYSTEM}

The research into agent-based intelligent decision support systems is important to the medical industry because these systems can be used to improve the quality of healthcare in many ways. The areas these systems can be used in are diverse from the storage of medical records to the examination and evaluation of real-time data gathered from monitors. These systems are helpful to doctors and nurses in the diagnosis and treatment of patient with all kinds of conditions.

Here we want to design such type of system such that the patients can access their previous clinical record on the internet, if a clinical data repository exists as shown in the figure.

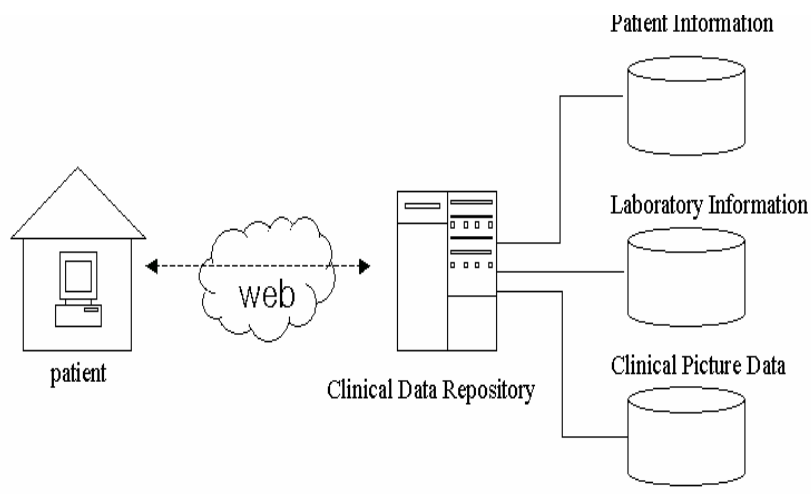

Figure4. : Patients can access their clinical history with a computer in home or office.

Further, an electronic healthcare record is a structured multimedia collection of health-care data about an individual patient. Extracts of individual patient records, obtained from a system and brought together in a structured format, as shown in the figure.

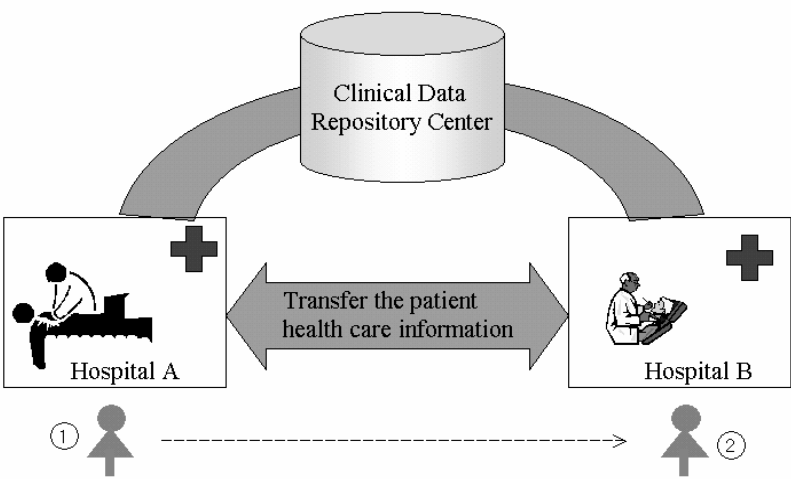

Fig.5

Health-care records are widely distributed and have strong local autonomy. So, for consistency, we adopt HL7 standards in the present project. The name HL7 reflects the seventh (application) level of the OSI reference model. The primary goal of HL7 is to provide a standard for the exchange of data among hospital-computer applications. The HL7 standard is message based and uses an event trigger model that causes the sending system to transmit a specified message to the receiving unit with a subsequent response by the receiving unit. 
So, we are supposed to design such type of system which can access medical records which are in distributed form using the HL7 interface.

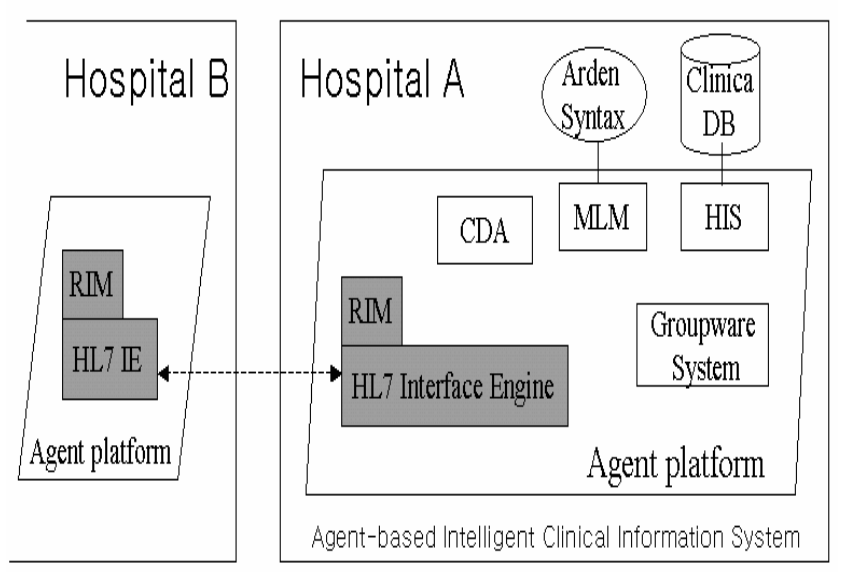

\section{Figure 6: Inter-hospital communication through the HL7 interface engine RIM}

To bridge this gap the project DEPR (Distributed Electronic Patient Record) is suggested \& the research will be carried out to formulate the methods $\&$ simulate the results. The proposed research DEPR project aims at integrating electronic medical record (EMR) data through the national computer network for health institutions ("HEALTHNET"). The DEPR project is expected to use advanced information and communication technology, including mobile agent computing.

The system will be based on EMR database formats. DEPR will be based on EMR patient data from both home-based caring service and for GPs.

\section{CONCLUSION}

The use of agent-based intelligent decision support systems to support decision making is important within the medical industry because they allow doctors and nurses to quickly gather information and process it in various ways in order to assist with making diagnosis and treatment decisions. For effective communication among the hospital or clinical institutions, sharing of clinical data records for decision support and providing the records to the patients, cooperative attitudes of common participants are needed. Surely there exist potential difficulties because many legacy systems are heterogeneous and the integration of these systems would not be easy.

The basic problem faced by many medical records vendor is of data extraction. This made the classic idea of research needs as to extract data in uniform ways and to store it on multi platforms in a distributed database environment is a great challenge.

With the proposed system the search \& indexing of EMR on any platform will be made with an ease \& the software medical service providers will be hugely benefited.

\section{REFERENCES}

[1] Amanda Ryan, "Towards Se mantic Inter operability in Healthcare: Ontology Mapping from SNOMED -CT to HL7 version 3", ACM Digital Library, vol 72, ISBN:1920-68253-8, Australia 2006.

[2] Surya Nepal, John Zic, Frederic Jaccard, Gregoire Kraehenbuehl, "A Trusted System for Sharing Patien Electronic Medical Records in Autonomous Distributed Health Care Systems", International Journal of Healthcare Information Systems and Informatics, Volume 2, No. 1, 2007.

[3] Muhammad Nabeel, "A secure online medical information system in distributed and heterogonous computing environment", International Relations \& Security Network Digital Library, Vol.15, No.2, P. No.: 211-215, 2004

[4] Muhammad Nabeel, "A secure online medical information system in distributed and heterogonous computing environment", International Relations \& Security Network Digital Library, Vol.15, No.2, P. No.: 211-215, 2004. 